\section{Antimicrobial Dosing Error in Intermittent \& Continuous Hemodialysis}

\author{
Saja M. Alhabardi ${ }^{*}$, Maha Almoliki ${ }^{2}$, Albatoul Almeshari ${ }^{1}$, and \\ Maha Alammari ${ }^{2}$ \\ ${ }^{1}$ The Saudi Food and Drug Authority, Riyadh, Saudi Arabia \\ ${ }^{2}$ King Abdulaziz Medical City- Central Region, Riyadh, Saudi Arabia
}

\begin{abstract}
Address for correspondence:
Saja M. Alhabardi, The Saudi Food and Drug Authority, Riyadh, Saudi Arabia. E-mail: ms.saja_m@yahoo.com.
\end{abstract}

How to cite this article: Alhabardi SM, Almoliki M, Almeshari A, and Alammari M. Antimicrobial Dosing Error in Intermittent \& Continuous Hemodialysis. G Med Sci. 2021; 2(3): 008-009.

https://www.doi.org/10.46766/thegms.intmed.21051603

Copyright: (c) 2021 Saja M. Alhabardi, Maha Almoliki, Albatoul Almeshari, and Maha Alammari. This is an Open Access article distributed under the Creative Commons Attribution License, which permits unrestricted use, distribution, and reproduction in any medium, provided the original work is properly cited.

\section{Background}

Antibiotic drugs are the most frequently prescribed medications among hospitalized patients for life-saving purposes, mainly in immunocompromised patient, like in patient with end stage renal disease on hemodialysis (HD) or those who had documented bacterial infection [1]. Antibiotics disposition and their pharmacokinetics and pharmacodynamics properties are affected in hemodialysis patient which increases the number of antibiotics dosing errors [2]. Several epidemiological studies found that the majority of medication error related to antibiotics occurred during the prescribing phase $(30.8 \%)[3,4]$. However, there is missing data about the prevalence of errors in antibiotics dosing in HD patients.

\section{Aim}

To evaluate the prevalence of errors in antibiotics dosing in HD patients.

\section{Objective}

To describe the most commonly prescribed antibiotics in HD patients, identify the most frequently occurring errors during prescribing the antibiotics in HD patients, which include the errors in dosage, or frequency.

\section{Method}

An observational retrospective cohort study included around 110 end-stage chronic kidney disease patients on HD over a one-year period (2016 to 2017). We collected the demographic data which included age, gender, height in $(\mathrm{cm})$, weight in $(\mathrm{kg})$, ideal body weight was calculated in patients who received aminoglycosides antibiotics or Colistin, and the antibiotic received including its dose, route, and frequency.

\section{Result}

From a total of $110 \mathrm{HD}$ patients were collected, male gender counted for $57.3 \%$ of them with an average age 63 years \pm 16 . Empirical antibiotics were administered to $73.64 \%$ HD patients. Cefazolin, piperacillin-tazobactam, ciprofloxacin, and amoxicillin-clavulanic acid were the most frequently prescribed antibiotics in HD patients $(26.4 \%, 16.4 \%, 13 \%$, and $13 \%)$, respectively. However, $27 \%(n=30)$ of HD patents did not receive an appropriately adjusted-dose antibiotics. Medication errors related to prescribing the antibiotics in HD patients mainly occurred with the Meropenem (86\%), by receiving it with wrong dose and frequency. Half of HD patients received an inappropriate ciprofloxacin and amoxicillin-clavulanic acid antibiotics regimen, mainly with oral route. The wrong frequency was reported with $71.43 \%$ among those who received an oral ciprofloxacin, and $57.14 \%$ in those who received oral amoxicillin-clavulanic acid antibiotic. 


\section{Conclusion}

The result showed that errors in prescribing antibiotics in HD patients are common. The errors mainly occurred with the prescription of meropenem, ciprofloxacin, and from amoxicillin-clavulanic acid antibiotics. This proves that enhancing health care providers' awareness about appropriate dosing regimens in $\mathrm{HD}$ patients is very importance.

\section{References}

1. Jon P. Furuno et al. Frequency of Outpatient Antibiotic Prescription on Discharge to Hospice Care. Antimicrobial Agents and Chemotherapy. 2014 sep; 58(9).5473-5477.
2. Heintz H. et al. Antimicrobial Dosing Concepts and Recommendations for Critically Ill Adult Patients Receiving Continuous Renal Replacement Therapy or Intermittent Hemodialysis. Pharmacotherapy 2009;29(5):562-577.

3. Briana B. Rider et al. Prescribing Errors that Cause Harm. Pennsylvania Patient Safety Advisory. 2016 sep; 13(3):81-91.

4. Bobb A et al. The epidemiology of prescribing errors: the potential impact of computerized prescriber order entry. Arch Intern Med. 2004 Apr 12;164(7):785-92. 